\title{
INTELLIGENT FAULT DETECTION DEVICE FOR WIND TURBINE IN REAL TIME CONDITION MONITORING
}

\author{
Saad CHAKKOR, Mostafa BAGHOURI, Abderrahmane HAJRAOUI \\ *University of Abdelmalek Essaâdi, Faculty of Sciences, Department of Physics, \\ Communication and Detection Systems Laboratory, \\ BP. 2121 M'Hannech II, 93030, Tetouan, Morocco \\ Email: saadchakkor@gmail.com, baghouri.mostafa@gmail.com, ad_hajraoui@hotmail.com
}

\begin{abstract}
Wind power has progressed nowadays because the proliferation of wind parks and their operation in supplying the national electric grid with low cost and clean resources. Hence, there is an increased need to establish a proactive maintenance for wind turbine machines based on remote control and monitoring which rely heavily on the timely delivery of safety real-time data for offshore or inaccessible locations through a wireless connection while the wired method has many flaws. The objective of this strategy is to prolong wind turbine lifetime and to increase productivity. The hardware of a remote control and monitoring system for wind turbines Park is designed by taking advantage of GPRS or Wi-Max wireless module to collect data measurements from different wind machines sensors through IP based multi-hop communication. Computer simulations with Proteus ISIS and OPNET tools have been conducted to evaluate the performance of the studied system. Study findings show that the designed device achieves a satisfactory performances and it is suitable for application in a wind park.
\end{abstract}

Keywords: Embedded System, Monitoring, Wind Turbine, Faults Diagnosis, TCP/IP Protocol, Real Time.

\section{INTRODUCTION}

In front of the huge increase demand in energy over the world, and in order to search a substitutional kind of energy against the prices growth of the energy fossil fuels resources and it exhaustion reserves in the long term, furthermore the commitment of the governments to reduce greenhouse gases emissions have favoured the research of others energy sources, the recourse to renewable energy becomes a societal choice. The development of this alternative is encouraged because it offers natural, economic, clean and safe resources. Among the renewable energies, wind energy which has been progressed in a remarkable way in these recent years. It provides a considerable electrical energy production with fewer expenses with exception of construction and maintenance budget. Actually, wind energy investment has increased by the multiplication of the wind parks capacities. This contributes greatly to the expansion of terrestrial and offshore wind parks. These parks are offshore or usually installed in far locations, difficult to access, subject to extreme environmental conditions [1]. Wind turbines contain a complex electromecanical system which is prone to defects. Therefore, their monitoring and diagnosis become essential to reduce maintenance costs and ensure continuity of production because stopping a wind installation for unexpected failures could lead to expensive repair and to lost production [2]. This operating stopping becomes critical and causes very significant losses. For these reasons, there is an increase need to implement a robust efficient remote maintenance strategy to ensure uninterrupted power in the modern wind systems [3]. This on line surveillance allow an early detection of mechanical and electrical faults preventing major component failures, facilitating a proactive response, anticipating the final shutdown of wind generators, minimizing downtime and maximizing productivity by analysis of measured physical signals continuously collected from different types of sensors [4], [5], [6]. This is why reliability of wind turbines becomes an important topic in scientific research and in industry. Most of the recent researches have been oriented toward electrical monitoring, as it would be the most practical technique and less costly. Another powerful tool used for diagnosis of an induction motor or generator utilizing the result of the spectral analysis of the stator current to indicate an existing or incipient failure is current stator analysis (CSA) [1], [4], [5], [6], [7]. Furthermore, with recent digital signal processor (DSP) and with wireless communication technology developments, it is possible to detect electric machines faults prior to possible catastrophic failure in real-time based on the stator line current allowing precise and low-cost [7]. The main object of this paper is to study the design of a real time monitoring and controlling system for condition supervision of wind generator machines which integrates intelligence and robustness functions.

\section{RELATED WORK}

In the literature review, few of research studies have been developed to analyze the theoretical aspects of the application part in condition monitoring of wind turbines operating [1], [8], [9], [16]. As known these faults cause a modulation impact in the magnetic field of the wind generator, which is reflected by the appearance of a significant harmonics (peaks) in the stator current spectrum [3]. Some research works are applying enhanced signal processing techniques like Fast Fourier Transform method (FFT), Short Time Fourier Transform (STFT), periodogram, Discrete Wavelet Transform (DWT), Wigner-Ville representation, Concordia Transform (CT) and the Hilbert-Huang transform and other advanced tools based on wind generator stator current to diagnose prospective electromechanical faults under transient conditions [3], [6], [14], [15]. Otherwise, in [2] a statistical diagnosis approach is proposed based on residues analysis 
of the electrical machine state variables by the use of the Principal Components Analysis method (PCA) for faults detection in offshore wind turbine generator. The aim drawback of this approach is that the detection efficiency requires a good choice of the principal components number. Some researchers are proposed failures diagnosis and monitoring of wind turbines generators using impedance spectroscopy (IS) [17-21]. Due mainly to their advantages, High resolution methods (HRM) such as MUSIC, ESPRIT and their zooming techniques having high accuracy can detect frequencies with low SNR [21], [22], [24]. They have been recently introduced in the area of induction motors and wind generators faults diagnosis to improve the detection of a large number of frequencies in a given bandwidth. Others practical research techniques are used to solve online maintenance problems with the help of real time by integration of the Wireless Sensor Network and the industrial communication protocols in wind turbines supervision and diagnosis [10], [11], [12], [13],[20], [23]. This paper presents an intelligent remote monitoring and fault diagnosis system for wind turbine using real time hardware based on a wireless communication module.

\section{PROBLEM FORMULATION}

The current maintenance planning based on-line monitoring systems used in wind parks is not optimized. It has several limitations both in terms of performance and in terms of installation cost. This is the results of various reasons:

- Cable structure monitoring with its problems (cuts, noise, and configuration), mainly through fiber optic, is not effective and not appropriate since wind parks are often deployed over a large geographic area having environmental obstacles such as offshore, desert, mountains, rivers, forests and plains that are located far away from the control center.

- Wind turbines monitoring need to implement a proactive maintenance system based on an early, fast and real time fault detection and diagnosis, allowing a secure and reliable communication for better maintenance management. This strategy avoids failures leading to serious damages, expensive repair and production loss.

- The storage of the monitored parameters in the control center database is a need, because it is an essential operation which helps to monitor accurately the lifetime of the wind turbine components. Therefore, it allows exploiting collected data for studying statistically the most occurring faults and their timing.

To overcome these imposed application constraints, an interactive embedded system has been designed to provide an efficient, reliable and economical link between various wind turbine sensors for an accurate remote controlling and monitoring scheme.

\section{MONITORED PARAMETERS IN WIND TURBINE}

Since the wind turbine machine is a complex system, it contains a large number of parameters to be monitored. For this reason to pursue and to controls all functions of the wind turbine in order to ensure it optimum operation at any wind velocity, there is a strong need to install a set of sensors continuously collecting performance data of the wind turbine and they transmit it to an internal controller which is designed to allow local supervision and command in case of the machine functions are required. In case of anomalies or errors, the data is stored in a database or log alarm, allowing error analysis of wind machine. The monitored parameters are acquired through ADC devices connected by means of the direct connection or by RS232 and USB interfaces of the computer or by a wireless connection. When a failure occurs inside the wind turbine, the controller reacts quickly to avoid the evolution of any type of defects. In this optic a robust and reliable system must be developed leading to the possibility of real time and detecting faults accuracy in their incipient stages before they transform into more serious failures causing undesirable downtimes and damages. The table 1 enclose the list of sensors and the parameters monitored in wind turbine.

Table 1 Monitored parameters

\begin{tabular}{|l|l|}
\hline \multicolumn{1}{|c|}{ Parameter } & \multicolumn{1}{c|}{ Sensor } \\
\hline $\begin{array}{l}\text { Bearing, gearbox } \\
\text { lubrication }\end{array}$ & SKF Windlub \\
\hline Windvane & INV-40A \\
\hline Anemometer & INA-46A \\
\hline Temperature, gear bearing & PT100 \\
\hline Temperature, generator & PT100 \\
\hline External Air Temperature & PT100 \\
\hline Temperature Nacelle & PT100 \\
\hline Temperature gear oil & PT100 \\
\hline Rotor speed (gear tooth) & EI 30 10 PPos, PNP \\
\hline Generator speed & DU 10, EP, PNP \\
\hline $\begin{array}{l}\text { Pitch position } \\
\text { (linear actuator) }\end{array}$ & Vert-X 22 \\
\hline Atmospheric Pressure & NRG \#BP20, WXT520 \\
\hline Humidity & P14 SMD \\
\hline Vibration & WLNJ-S2-G, HS-100 \\
\hline Hydraulic oil level & Gems LS600-150-NO-10 \\
\hline
\end{tabular}

Figure1 gives a detailed description of the location of the various sensors used in the monitoring of a wind machine. The current/voltage intelligent sensors, based on generator current stator spectral analysis (CSA), are used in cooperation with one bit vibration sensors for an early identifying of prospective occurring mechanical faults.

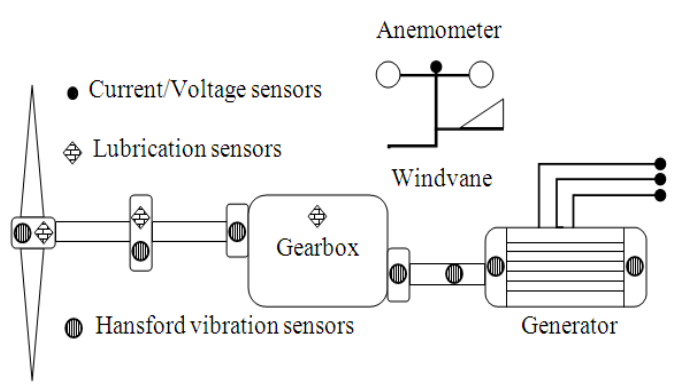

Fig. 1 Sensors localization scheme 


\section{DESIGN OF SYSTEM STRUCTURE AND NETWORK}

The proposed system is an Intelligent Embedded System for Control and Remote Monitoring (IESCRM) that has the capability of processing, monitoring and controlling [29], this system finds its application in many field areas especially remote electromechanical condition monitoring of wind turbines in a park. It comprises sensors, microcontroller, memories, etc. It typically has a specialized function with programs stored on ROM. An added feature in this embedded system is its ability to communicate. The communication can be via Wi-Fi, GPRS, Wi-Max or Ethernet cables. The TCP/IP protocol is a widely used standard for modern digital communication. It provides real time data traffic. The main goal of this hardware is to make internet/IP enabled for the developed embedded device which serving as advanced remote data logger to be accessed remotely via workstation. The mentioned device will be interfaced with various sensors as illustrated in table 1.

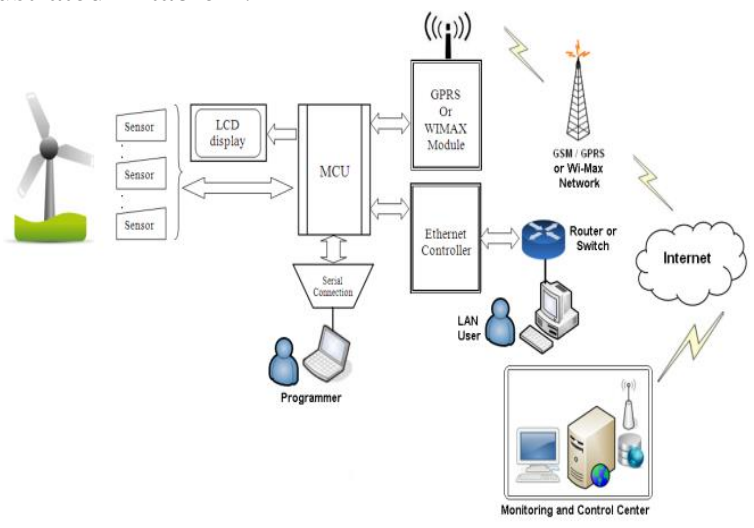

Fig. 2 Block diagram of monitoring and control system Architecture

The measurements and control data are then communicated to the central server, which adopts client/server web and database frameworks, through an Ethernet or wireless connection. This server holds all the past data transmitted by the system using web pages. Figure2 illustrate the monitoring and control system architecture allowing on-line data transfer between the wind turbines and the control center. The designed interactive embedded hardware provides an efficient, reliable and economical link between various systems, sensors and actuators. The choice of the GPRS and Wi-Max protocols is justified by several reasons: first, the wind parks are implemented on a large geographical area that reaches some kilometers, thereafter their remote monitoring requires a wide cover wireless communication protocol, then the implementation of these communication technologies does not require any new infrastructure installation because it uses the mobile cellular telephony network $2.5 \mathrm{G}, 3 \mathrm{G}$ or $4 \mathrm{G}$. Some differences existing between these two protocols are data rate and transmission time [25].

The sensors listed in table 1, are connected to the microcontroller which is used as a computer and the Ethernet chip ENC28J60 [28] is used to connect the microcontroller to LAN with Ethernet cable by a RJ45 port. Figure 3 show the interconnection of ENC28J60 with MCU.
The electrical power supply of the IESCRM module does not pose a problem because it can be powered by a rechargeable battery connected to an $\mathrm{AC} / \mathrm{DC}$ conversion circuit of the current received from wind turbine during operation.

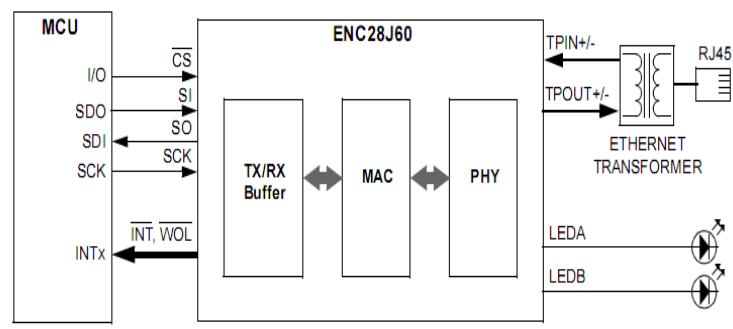

Fig. 3 Ethernet Chip and MCU interconnection

Whereas, Wi-Max or GPRS module (like ZTE IX256-RJ45 Modem [26] for Wi-Max or SIM900A-RS232 Modem [27] for GPRS) are used to establish an on-line wireless internet connection with the base station server. The sensors are connected to the $\mathrm{AN}_{0-7}$ pins. The $\mathrm{MCU}$ is chosen from PIC24 family of microcontrollers because it has many advantages: large number of I/O pins, high memory space, built in ADC channels which make it easy for interfacing the sensors, developed using NanoWatt technology that reduces power consumption during operation. The LCD displays the current and any new IP address of the communication. The serial connection RS232 is used to configure the module (to change the IP address). It can also be used for debugging purposes. The IESCRM module is an automaton with integrated web server. Once programmed, it contains software consisting of web pages for configuring, monitoring and visualization of data measurements of the differents sensors connected to this module. Both GPRS and Wi-Max are two IP based service protocols which can be used to access a range of IP services by running TCP/IP protocol stack, such as FTP, Web Browsing, Machine-to-Machine M2M and Simple Mail Transfer E-mail SMTP. TCP/IP Stack is divided into multiple layers. The code implementing each layer resides in a separate source file, while the services and APIs (Application Programming Interfaces) are defined through header/include files. Another protocol used in IESCRM module is HTTP which simply involves an exchange of text messages followed by the transfer of Web data via a TCP connection. To fetch a Web page, the browser opens a TCP connection to server port 80, and then uses HTTP to send a request. The basic program which is localized in MCU EPROM runs as a server providing multi-user access to a number of databases. A MySQL/PHP Web/Database server based on APIs are used to implement adequate software through CGI protocol for interfacing external application software with an information web server. By this technique, the information regarding physical parameters is sensed from the sensors. This is received by microcontroller and is stored in CGI variables. These CGI variables can be accessed by CGI scripts running in web/database server. The data are stored in the MySQL database. Finally the required graphical representation of the data can be displayed interactively in the dynamic website as illustrated in figure4. The developed module offers several features that require a SIM card:

- Access to web pages of the module via Ethernet 
connection, GPRS or Wi-Max

- Sending alarm messages by e-mail via GPRS or Wi-Max

- Sending alarm messages by SMS

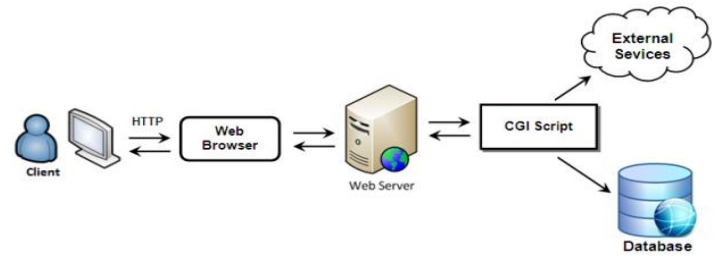

Fig. 4 CGI and Web Server connection with Database

The IESCRM module provides an early damage detection procedure that enables remote, automatic maintenance and control of a wind turbine hard to access, eliminating the need for an on-site service engineer to perform extra manual intervention. Reacting to the problems detected by this system. A condition monitoring specialist can set the proper alarm settings to trigger appropriate decision. If failures are detected such as empty or blocked lubrication pumps or torn feed lines or oil/grease levels, operators are notified immediately. In this case, depending on the type of fault and it severity, it will be necessary that the wind turbine controller take an operating strategy of around four operational states:

- On

- Pause

- Off

- Emergency

\section{CIRCUIT DESIGN USING PROTEUS SOFTWARE}

To evaluate the operation of the designed module, it computer simulation has been made with Proteus ISIS software version 7.7, figure5 show electronic schematics circuits of IESCRM with a GPRS module. Furthermore, the figure6 present electronic schematic circuits of the IESCRM using Wi-Max modem. The architecture of the centralized network for supervision and remote maintenance of a wind park can be described as shown in figure7.

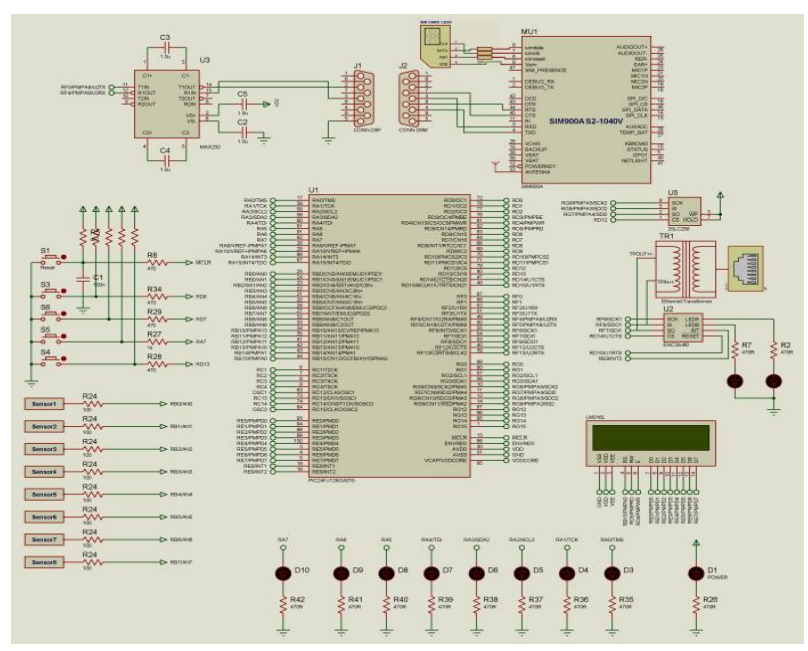

Fig. 5 Electronic schematics of IESCRM with GPRS module
The web pages can be viewed by browsing directly to the specific IP address device from the base station or from any other computer on the network. To maximize program memory space (and be able to fit the code in a PIC24FJ128GA010) an external 25LC256 Serial EEPROM is added which uses the SPI interface that can be shared with the ENC28J60 and the device much faster than the $\mathrm{I} 2 \mathrm{C}$ part.

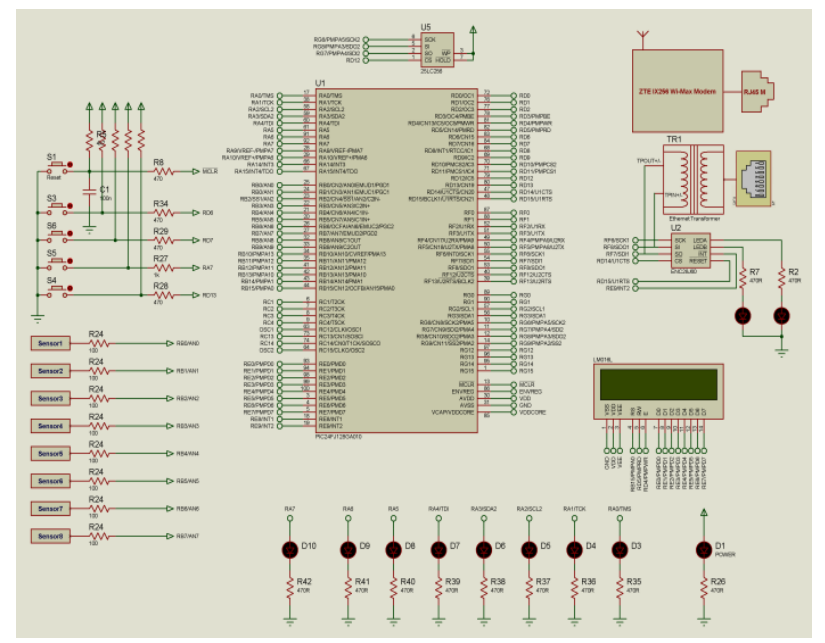

Fig. 6 Electronic schematics of IESCRM with Wi-Max modem

The IESCRM physical interface model transmits and receives data packets to base station through GPRS or WiMax connection. Meanwhile, the PIC24 runs it own TCP/IP stack and software, comprising a simple web server. This software enables the microcontroller to behave as a communications server, providing a support for a web client/server. When the simulation is started, the LCD display the IP address allocated to the wireless modem by the office network DHCP server. For demonstration purpose the IP address of different IESCRM in wind park network are assigned to be 192.168.11.X, where $\mathrm{X}$ take it value between 2 to 255 and the IP of the central web/database server was assigned to be 192.168.1.1, both connected to same network router.

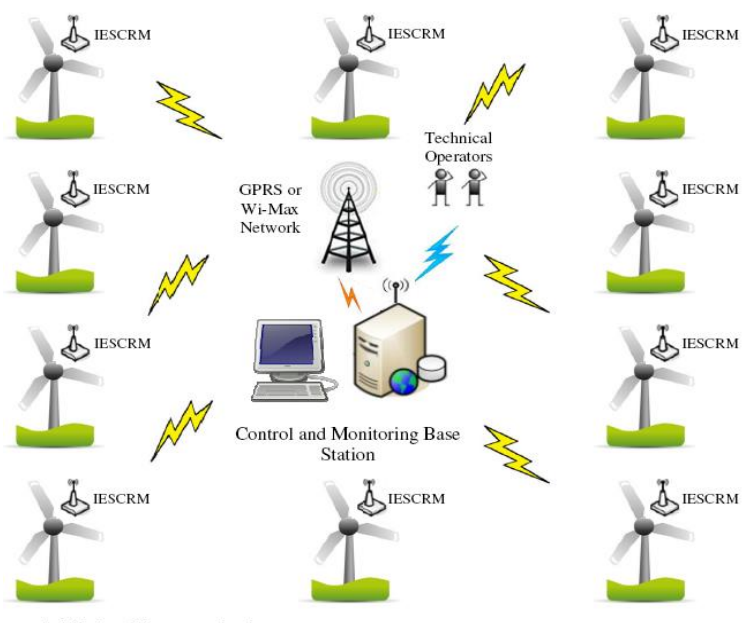

Fig. 7 Structure of control and monitoring system of a wind park 
In this architecture, each wind machine is equipped with an IESCRM module identified by its own IP address, this allows to collect and to store data received from different sensors installed on each wind turbine.

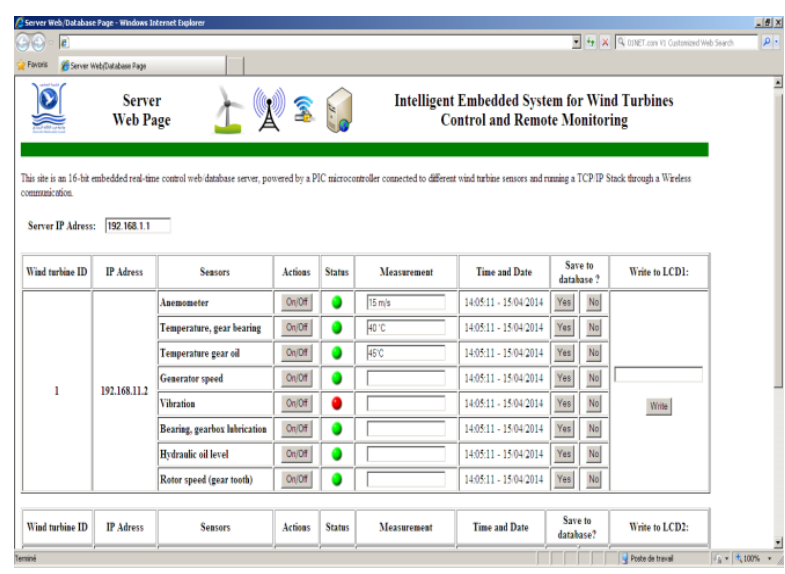

Fig. 8 IESCRM Server Web/Database Page monitoring

The IESCRM takes care of the real time regular transmission of all measurements via GPRS or Wi-Max to the server of supervision and control station which has a specific IP address. This communication is established by means of a dynamic web page showed in figure 8 indicating the time and date, the wind turbine ID and it IP address and the received data. On the other side, IESCRM collaborate with the central automaton regarding the operation mode of the wind machine. The base station server allows management and processing of the database samples received from different wind turbines in order to make a decision or to apply a command using diagnostic algorithms.

\section{SIMULATION RESULTS}

The system described in the previous section for wind park controlling and monitoring has been implemented and simulated based on a realistic task by the OPNET Modeler 14.5 an Proteus ISIS via virtual serial connection ports under two scenarios and carried out the simulation to evaluate and to compare the performance of the IESCRM system in term of real time data traffic for two different network technologies. The scenarios developed in OPNET for Wi-Max and GPRS over UMTS are shown in figure 9 and 10 and the system parameters for both networks are detailed in table 2 and 3 .

Table 2 Wi-MAX Simulation Environment Parameters

\begin{tabular}{|l|l|}
\hline \multicolumn{2}{|c|}{ System Parameters } \\
\hline Simulation time $(\mathrm{sec})$ & 3600 \\
\hline Data traffic rate & $10 \mathrm{Mbps}$ \\
\hline Basic rate & $1.5 \mathrm{Mbps}$ \\
\hline Service Class Name & Gold/UGS \\
\hline Antenna Gain & $15 \mathrm{dBi}$ \\
\hline PHY profile & Wireless OFDMA 20 MHz \\
\hline Max. Transmit power & 0.5 Watt \\
\hline Path loss & Pedestrian \\
\hline BS MAC address & Distance based \\
\hline
\end{tabular}

Table 3 UMTS Simulation Environment Parameters

\begin{tabular}{|l|l|}
\hline \multicolumn{2}{|c|}{ System Parameters } \\
\hline Simulation time (sec) & 3600 \\
\hline UMTS MN cell state & CELL_DCH \\
\hline UMTS RLC process time & $0.015 \mathrm{sec}$ \\
\hline CPICH transmission Power & $1 \mathrm{Watt}$ \\
\hline Shadow fading Standard deviation & 10 \\
\hline Processing time & $0.02 \mathrm{sec}$ \\
\hline Path loss & Pedestrian \\
\hline UMTS GMM Timer & $15 / 30 / 10$ \\
\hline
\end{tabular}

Two different applications are used: HTTP and Database. The studied Wi-MAX and UMTS network models consists of two Base Stations and two cells; each cell has four fixed wind turbine machines which are equipped with an IESCRM to serve all applications types. The coverage of one cell is approximately $2 \mathrm{~km}$ by $2 \mathrm{~km}$ of area

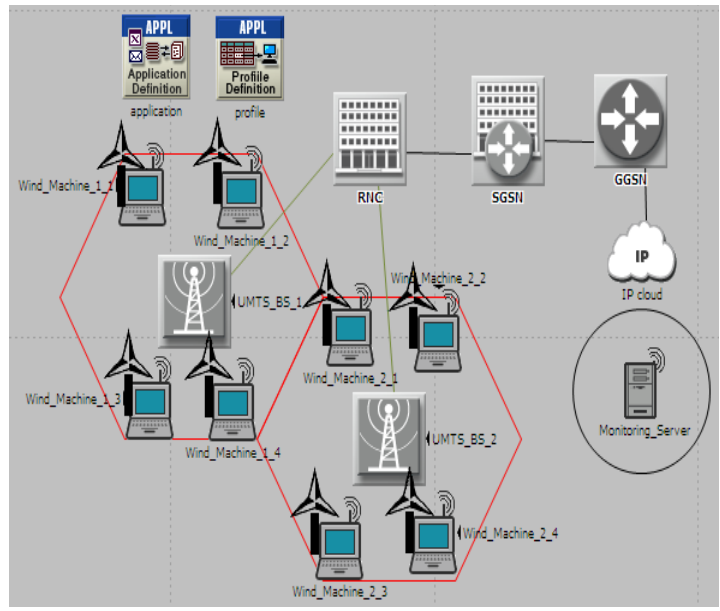

Fig. 9 GPRS over UMTS wind park monitoring OPNET scenario

UMTS model shown in figure 9 comprises user equipments, Radio Network Controller (RNC) which is connected to the packet switched network via GPRS Support Node (SGSN) and through GPRS Gateway Support Node (GGSN) which in turn is connected to the IP Network.

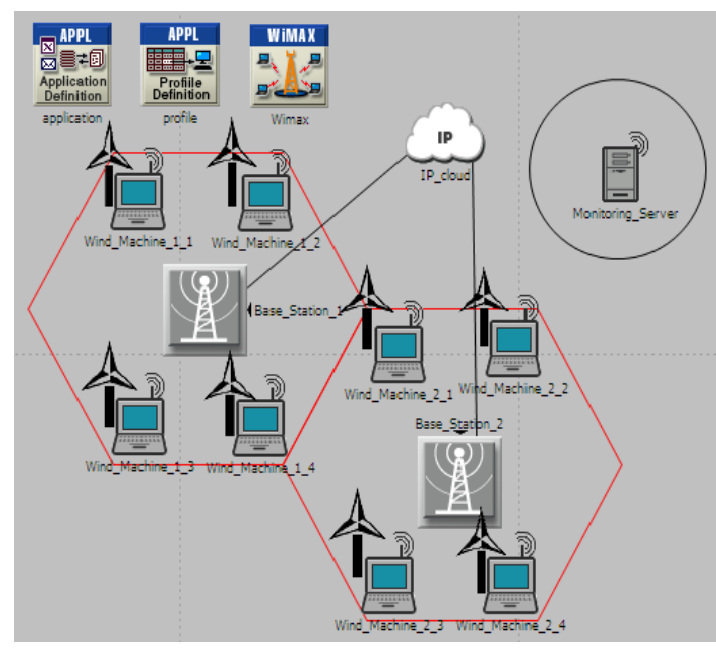

Fig. 10 Wi-Max wind park monitoring OPNET scenario 
Based on figure 11, the average response time in database query for GPRS has an unstable behavior. It is delayed with almost 127 times compared to the same time for Wi-Max network. In other side, it is clear from figure 12 that the average traffic received in database query increase significantly with time increasing and it takes a fixed maximum value for both GPRS and Wi-Max networks.

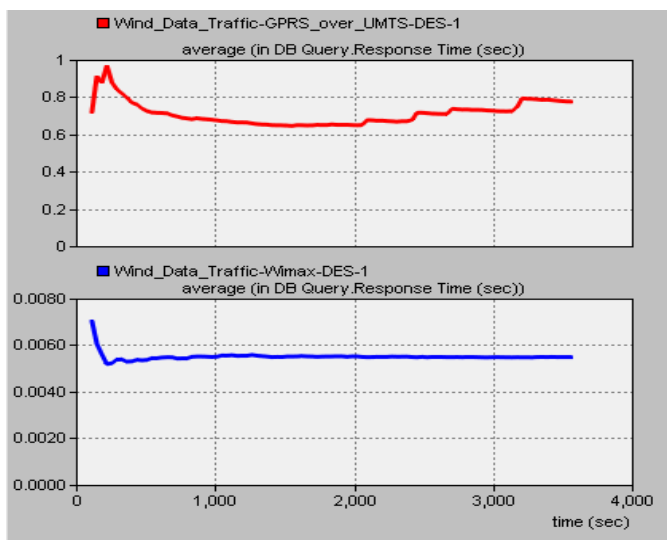

Fig. 11 Average response time in Database Query

The traffic received is much more important for the WiMax compared to GPRS and the difference between them is about $80 \%$.

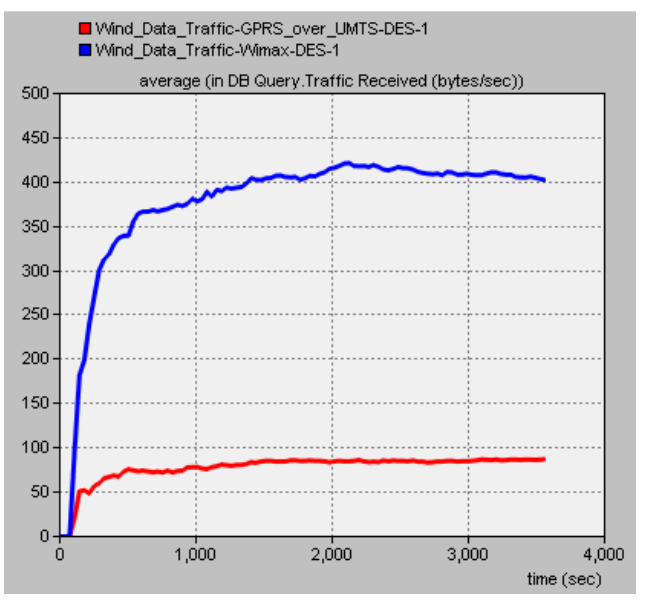

Fig. 12 Average traffic received in Database Query

Whereas, referring to the simulation results illustrated in figure 13, Wi-Max is very faster to provide web page in HTTP service and it exceeds GPRS with about 187 times in average. This page response time is has an unstable evolution. Concerning the average traffic received in HTTP service for GPRS and Wi-Max it shown in figure 14, it can be observed that the value of this parameter is greater for Wi-Max than GPRS with about 94\%. Indeed, as illustrated in figure 15, average network activation delay for Wi-Max is multiplied 137 times than that of GPRS. The obtained results can be justified by the fact that GPRS network covers a wide area but it data transmission is only able to achieve a data rate between $35 \mathrm{Kbit} / \mathrm{s}$ and $87 \mathrm{Kbit} / \mathrm{s}$ in practice. This is much less than Wi-Max which can reach $75 \mathrm{Mbit} / \mathrm{s}$. Wi-Max is able to bring more bandwidth and provides more connection services quality to benefit in the wind park monitoring.

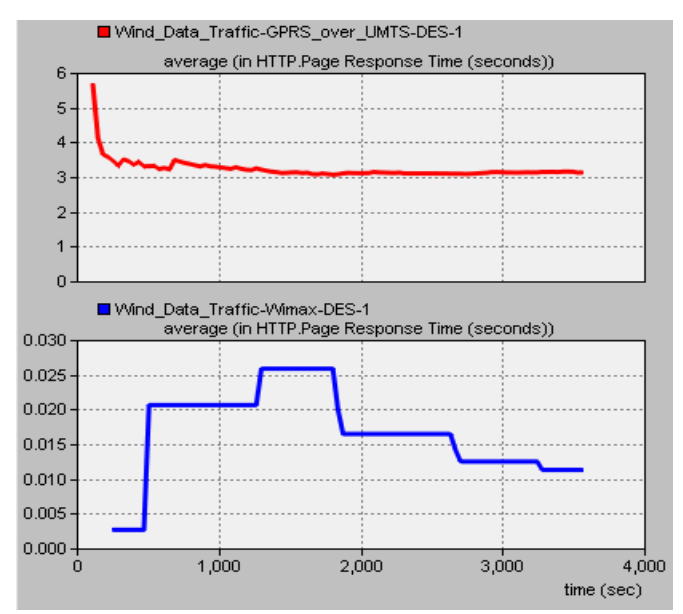

Fig. 13 Average Page response time in HTTP service

Furthermore GPRS has a huge fluctuation in the speed whereas Wi-Max gives a constant bandwidth which resulting a constant and a fastest Internet service.

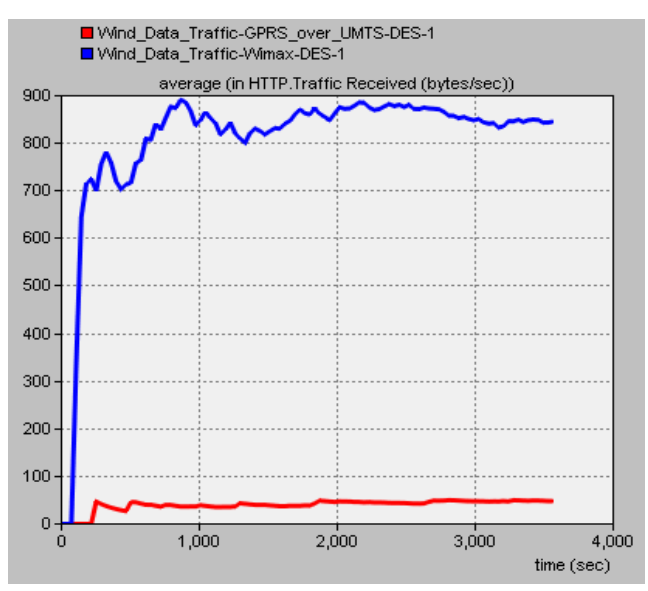

Fig. 14 Average traffic received in HTTP service

Wi-Max remains the most appropriate technology choice to satisfy the QoS requirements and real time data traffic for wind turbines control and monitoring application.

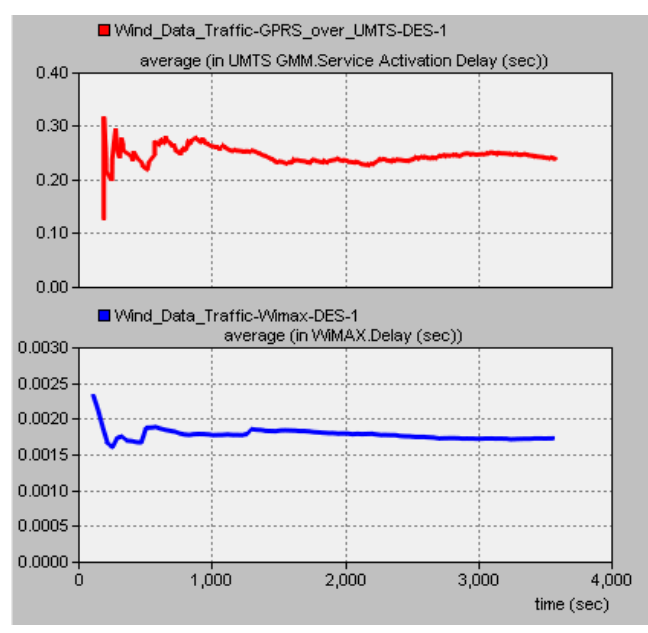

Fig. 15 Average network activation delay 


\section{CONCLUSIONS}

Wind turbine machines needs periodical proactive maintenance to prolong their electromecanical components lifetime. The main contribution of this paper is to design by study and analysis an intelligent wireless remote monitoring and control system according to wind turbines features and requirements. Both hardware module and operating mode have been described in detail. This system based on IP communication combines Web and database client/server technology to copy data measurements received from the differents sensors installed in the wind turbine machines and therefore sends it to the base station through a wireless connection. The simulation comparison, with Proteus ISIS and OPNET simulators between GPRS and Wi-Max implementation in the studied module, favourites Wi-Max network due to their benefits over GPRS network. This intelligent device has low cost and it can be used in other application areas. Maintenance issues are now able to be resolved with this efficient communicant tool. The future work will be focused on the test and application of the whole monitoring system in the practice.

\section{REFERENCES}

[1] SIN, M. L. - SOONG, W. L. - ERTUGRUL, N.: “OnLine Condition Monitoring and Fault Diagnosis - a Survey" Australian Universities Power Engineering Conference, New Zealand 2003

[2] BENNOUNA, O. et al.: "Condition Monitoring \& Fault Diagnosis System for Offshore Wind Turbines",https://zet10.ipee.pwr.wroc.pl/record/425/f iles/invited_paper_3.pdf

[3] AL-AHMAR, E. et al.: "Wind Energy Conversion Systems Fault Diagnosis Using Wavelet Analysis", International Review of Electrical Engineering 3, 4 2008, pages: 646-652

[4] CHAKKOR, S. - BAGHOURI, M. - HAJRAOUI, A.: "ESPRIT Method Enhancement for Real-time Wind Turbine Fault Recognition", International Journal of Power Electronics and Drive System (IJPEDS), IAES Publisher, Volume 5, No 4, April 2015

[5] PANDEY, K. K. et al.: "Review on Fault Diagnosis in Three-Phase Induction Motor", MEDHA - 2012, Proceedings published by International Journal of Computer Applications (IJCA)

[6] AL AHMAR, E. et al.: “Advanced Signal Processing Techniques for Fault Detection and Diagnosis in a Wind Turbine Induction Generator Drive Train: A Comparative Study", IEEE Energy Conversion Congress and Exposition (ECCE), Atlanta: États-Unis 2010

[7] HAMID, A. TOLIYAT et al.: "Electric Machines Modeling, Condition Monitoring, and Fault Diagnosis", CRC Press Taylor \& Francis Group NW 2013, ISBN-13: 978-1-4200-0628-5

[8] HYERS, R. W. et al.: "Condition monitoring and prognosis of utility scale wind turbines", Institute of Materials, Minerals and Mining and W. S. Maney \&
Son Ltd Energy Materials 2006, Vol.1 No.3, pages $187-203$

[9] NILSSON, J. et al.: "Maintenance Management of Wind Power Systems Using Condition Monitoring Systems-Life Cycle Cost Analysis for Two Case Studies", IEEE Transactions On Energy Conversion, Vol. 22, No. 1, March 2007

[10] MOHANRAJ, M. et al.: “A CAN Bus based system for monitoring and fault diagnosis in Wind Turbine", International Conference on Emerging Trends in VLSI, Embedded System, Nano Electronics and Telecommunication System (ICEVENT), Tiruvannamalai, 7-9 Jan 2013, pages 1-3

[11] CHUN-LIANG HSU: "Constructing transmitting interface of running parameters of small-scaled windpower electricity generator with WSN modules", Expert Systems with Applications Journal 37, Elsevier 2010, pages 3893-3909

[12] ZAHER, A. et al.: "Database Management for High Resolution Condition Monitoring of Wind Turbines", Proceedings of the 44th International Universities Power Engineering Conference (UPEC), Glasgow 14 Sept. 2009

[13] MALLIKARJUNE GOWDA, M. C. et al.: "Improvement of the Performance of Wind Turbine Generator Using Condition Monitoring Techniques", Proceedings of 7 th International Conference on Intelligent Systems and Control (ISCO 2013), IEEE 2012

[14] AMIRAT, Y. et al.: "Wind Turbines Condition Monitoring and Fault Diagnosis Using Generator Current Amplitude Demodulation", IEEE International Energy Conference and Exhibition (EnergyCon), 2010

[15] MIHET POPA, L. et al.: "Condition Monitoring of Wind Generators", IEEE Industry Applications Society 38th Annual Meeting, IAS'03, Salt Lake City, Utah USA, October 2003, Vol. 3 IEEE Signal Processing Society, p.1839-1846.

[16] CRABTREE, CH. J.: "Survey of Commercially Available Condition Monitoring Systems for Wind Turbines", Durham University School of Engineering and Computing Sciences, 2nd November 2010, Revision: 05

[17] SHENG, S. - VEERS, P.: "Wind Turbine Drive train Condition Monitoring - an Overview", Mechanical Failures Prevention Group: Applied Systems Health Management Conference Virginia Beach, Virginia May $10-12,2011$

[18] XIANG GONG: "On-line Non-Intrusive Condition Monitoring and Fault Detection for Wind Turbines", DigitalCommons@University of Nebraska - Lincoln

[19] KLUGE, M. - DANITSCHEK, M.: "Condition Monitoring Systems (CMS) in wind turbines", ifm electronic gmbh, update version: 22.07.2010

[20] TUOMINEM, JUHA: "Hydraulic Boom Monitoring With IEEE 802.11 Based Wireless Sensors Network", 
Master of Science Thesis, Tampere University of Technology, October 2009

[21] AHMED, I et al.: "A Baseline Study for On-Line Condition Monitoring of Induction Machines", Australasian Universities Power Engineering Conference (AUPEC), 26-29 September 2004, Brisbane, Australia

[22] CRABTREE, C. J. et al.: "Detecting Incipient Wind Turbine Gearbox Failure: A Signal Analysis Method for On-line Condition Monitoring”, European Wind Energy Conference (EWEC), Warsaw 19 April 2010

[23] CHUNMING WU - LIANG CHENG: "The Monitoring System for the Wind Power Generation Based on the Wireless Sensor Network", International Conference on Future Electrical Power and Energy Systems 2012, Energy Procedia 17, p. 1020 - 1027

[24] Asif Saeed, "Online Condition Monitoring System for Wind Turbine Case Study", Master Thesis, University of Kalmar, Blekinge Institute of Technology, October 2008

[25] CHAKKOR, S. et al.: "Comparative Performance Analysis of Wireless Communication Protocols for Intelligent Sensors and Their Applications", International Journal of Advanced Computer Science and Applications, SAI Publisher, Volume 5 No 4, May 2014

[26] “ZTE IX256 WiMAX MODEM-User Manual”, ZTE Corporation, Aug. 2010, Version: V1.0

[27] “SIM900 GSM/GPRS RS232 Modem-User Manual”, Rhydo Technologies (P) Ltd, Dec.2011

[28] "ENC28J60Data Sheet", Microchip Technology Inc. 2006, DS39662B

[29] JEREMY BENTHAM, "TCP/IP Lean Web Servers for Embedded Systems", Second Edition, CMP Books Lawrence, Kansas 66046, Berkeley, California 2002
Received July 20, 2014, accepted March 20, 2015

\section{BIOGRAPHIES}

Saad Chakkor was born in Tangier Morocco. He's a member in the Physics department, Communication and detection Systems laboratory, Faculty of sciences, University of Abdelmalek Essaâdi, Tetouan Morocco, and his research area is: wireless intelligent sensors and theirs applications, frequency estimation algorithms for faults detection and diagnosis system in electromecanical machines. He obtained the Master's degree in Electrical and Computer Engineering from the Faculty of Sciences and Techniques of Tangier, Morocco in 2002. He graduated enabling teaching computer science for secondary qualifying school in 2003. In 2006, he graduated from DESA in Automatics and information processing at the same faculty. He works as teacher of computer science in the high school.

Mostafa Baghouri was born in Tangier Morocco. He's a member in the Physics department, Communication and detection Systems laboratory, Faculty of sciences, University of Abdelmalek Essaâdi, Tetouan Morocco, his research area is: routing and real time protocols for energy optimization in wireless sensors networks. He obtained a Master's degree in Electrical and Computer Engineering from the Faculty of Science and Technology of Tangier in Morocco in 2002. He graduated enabling teaching computer science for secondary qualifying school in 2004. In 2006, he graduated from DESA in Automatics and information processing at the same faculty. He work teacher of computer science in the high school.

Abderrahmane Hajraoui is a professor of the Higher Education at University of Abdelmalek Essaâdi. He's a director thesis in the Physics department, Communication and detection Systems laboratory, Faculty of sciences, University of Abdelmalek Essaâdi, Tetouan, Morocco. His research areas are: Signal and image processing, automation systems, simulation systems, antennas and radiation, microwave devices and intelligent wireless sensors networks. 